\title{
Massive and low-mass protostars in massive "starless" cores
}

\author{
Thushara Pillai ${ }^{1,2}$, Jens Kauffmann ${ }^{1,3}$, Qizhou Zhang ${ }^{4}$, Patricio Sanhueza ${ }^{5}$, Silvia Leurini ${ }^{1,6}$, \\ Ke Wang ${ }^{7}$, T. K. Sridharan ${ }^{4}$, and Carsten König ${ }^{1}$
}

\author{
1 Max-Planck-Institut für Radioastronomie, Auf dem Hügel 69, 53121 Bonn, Germany \\ e-mail: tpillai.astro@gmail.com \\ 2 Institute for Astrophysical Research, 725 Commonwealth Ave, Boston University Boston, MA 02215, USA \\ 3 Massachusetts Institute of Technology, 99 Millstone Road, Haystack Observatory, Westford, MA 01886, USA \\ ${ }^{4}$ Harvard-Smithsonian Center for Astrophysics, 60 Garden Street, Cambridge, MA 02138, USA \\ 5 National Astronomical Observatory of Japan, National Institutes of Natural Sciences, 2-21-1 Osawa, Mitaka, \\ Tokyo 181-8588, Japan \\ ${ }^{6}$ INAF - Osservatorio Astronomico di Cagliari, Via della Scienza 5, 09047 Selargius (CA), Italy \\ 7 European Southern Observatory, Karl-Schwarzschild-Str. 2, 85748 Garching bei Munchen, Germany
}

Received 31 December 2017 / Accepted 14 December 2018

\begin{abstract}
The infrared dark clouds (IRDCs) G11.11-0.12 and G28.34+0.06 are two of the best-studied IRDCs in our Galaxy. These two clouds host clumps at different stages of evolution, including a massive dense clump in both clouds that is dark even at 70 and $100 \mu \mathrm{m}$. Such seemingly quiescent massive dense clumps have been speculated to harbor cores that are precursors of high-mass stars and clusters. We observed these two "prestellar" regions at $1 \mathrm{~mm}$ with the Submillimeter Array (SMA) with the aim of characterizing the nature of such cores. We show that the clumps fragment into several low- to high-mass cores within the filamentary structure of the enveloping cloud. However, while the overall physical properties of the clump may indicate a starless phase, we find that both regions host multiple outflows. The most massive core though $70 \mu \mathrm{m}$ dark in both clumps is clearly associated with compact outflows. Such low-luminosity, massive cores are potentially the earliest stage in the evolution of a massive protostar. We also identify several outflow features distributed in the large environment around the most massive core. We infer that these outflows are being powered by young, low-mass protostars whose core mass is below our detection limit. These findings suggest that low-mass protostars have already formed or are coevally formed at the earliest phase of high-mass star formation.
\end{abstract}

Key words. stars: formation - ISM: clouds - ISM: jets and outflows

\section{Introduction}

An outstanding puzzle in the formation of high-mass stars is their earliest phase. What are the initial conditions of high-mass star formation? A frequently invoked theoretical concept is the turbulent core model (McKee \& Tan 2003) that predicts the onset of star formation from a monolithic, high-mass, starless dense core supported by turbulence. However, despite the technical advances in observing techniques and large surveys spanning the relevant energy spectrum, high-resolution $(<0.1 \mathrm{pc})$ observations have not been successful in confirming a signgificant population of such cores due to (1) their possible absence in massive prestellar clumps (Sanhueza et al. 2017) and (2) clear association with molecular outflows (Bontemps et al. 2010; Pillai et al. 2011; Wang et al. 2011; Lu et al. 2015; Tan et al. 2016). We note, however, that there is only a handful of interferometric studies on potential sites to find high-mass prestellar cores and Atacama Large Millimeter/submillimeter Array (ALMA) will play a key role in the near future.

The identification of such high-mass starless cores is a daunting task in the heavily clustered, typically distant ( $>1 \mathrm{kpc})$, highmass star-forming regions. One of the main observational criteria for lack of star formation adopted by observers is the absence of an infrared (IR) point source at 24 or $70 \mu \mathrm{m}$. Spitzer and particularly Herschel Space Observatory have been crucial in distinguishing the starless cores from the more evolved protostellar cores. However, limited linear resolution at kiloparsec distances combined with high extinction can significantly confuse the interpretation of IR emission (or lack thereof) in high-mass starforming regions. Therefore, such studies have been combined with astrochemical indicators of very cold $(<20 \mathrm{~K})$ conditions such as a high deuterium fractionation and depletion (Chen et al. 2010; Fontani et al. 2011; Pillai et al. 2011; Tan et al. 2013; Kong et al. 2016). However, how reliable are such indirect indicators? Can high extinction hide very young and low-luminosity protostars within such seemingly starless clumps? If so, this questions the existence of a high-mass starless stage in highmass star formation. Are the existing cases of high-mass starless cores really starless?

In this work, we report Submillimeter Array (SMA) $230 \mathrm{GHz}$ continuum and CO 2-1 line observations towards two high-mass $70 \mu \mathrm{m}$ dark clumps, G10.99-0.08 and G28.33+0.07. These clumps are embedded in two of the most well-studied infrared dark clouds (IRDCs), G11.11-0.12 at a distance of $3.6 \mathrm{kpc}$ (Pillai et al. 2006a; Henning et al. 2010; Kainulainen et al. 2013; Wang et al. 2016) and G28.34+0.06 at a distance of $4.8 \mathrm{kpc}$ (Wang et al. 2008; Butler \& Tan 2012; Ragan et al. 2012). These IRDCs host clumps at different stages of evolution (Zhang et al. 2009, 2015; Henning et al. 2010; Wang et al. 2011, 2014; Ragan et al. 2012) and our targets are the youngest massive dense clumps in both clouds that are dark even at 70 and $100 \mu \mathrm{m}$. At a distance of $<5 \mathrm{kpc}$, as shown below, we estimate masses 
$>500 M_{\odot}$ within $0.6 \mathrm{pc}$ (radius) of the peak dust continuum emission, with total bolometric luminosity $<24 L_{\odot}$. The dark cloud core G28.33+0.07 has been recently extensively studied at millimeter $(\mathrm{mm})$ wavelengths at high resolution, revealing two compact, collimated outflows from the brightest continuum object, which also shows evidence for significant CO depletion and deuteration in $\mathrm{N}_{2} \mathrm{D}^{+}$, all indicators of a very young core (Chen et al. 2010; Feng et al. 2016; Tan 2016; Kong et al. 2018). No high-resolution observations of G10.99-0.08 have been reported yet. Here, we present SMA high-resolution observations of G10.99-0.08 and G28.33+0.07 focusing on the outflow properties based on $\mathrm{CO} 2-1$ both towards and in the larger vicinity (within $0.6 \mathrm{pc}$ radius) of the densest clumps.

\section{Observations}

The SMA ${ }^{1}$ observations were made as part of a SMA minisurvey of high-mass starless core candidates at $230 \mathrm{GHz}$. The SMA observations were made with all eight antennae in the most compact configuration (sub-compact) in two tracks in September 2008. The observations were done in track sharing mode with several sources per track. The $230 \mathrm{GHz}$ receiver was tuned to the CO (2-1) line in the spectral band s13 of the upper side band (USB). We used a non-uniform correlator configuration to provide a higher spectral resolution of $\sim 0.2 \mathrm{~km} \mathrm{~s}^{-1}$ for the chunks covering specific spectral lines including CO 2-1. The rest of the correlator was set to $\sim 0.4 \mathrm{~km} \mathrm{~s}^{-1}$. The data were taken under very good weather conditions ( $<1 \mathrm{~mm}$ precipitable water vapor), and the typical system temperatures were $100 \mathrm{~K}$. The primary gain calibrator was $\mathrm{J} 1733-130,<12^{\circ}$ away from both targets. Uranus and 3C454.3 were used as the flux and bandpass calibrator, respectively. Flux calibration is based on the model of the observed planet and is estimated to be accurate to within $20 \%$. The primary beam at $230 \mathrm{GHz}$ is $\sim 51^{\prime \prime}$. The data calibration was done in MIR, an IDL-based calibration package, and then exported to MIRIAD and CASA for further data reduction, which included continuum subtraction and imaging. The center of the final image of the mosaic, the $1 \sigma$ rms noise level achieved in the continuum images, and the resulting synthesized beam for all observations are listed in Table A.1. For G10.99-0.08 CO 2-1 emission, we combine our sub-compact configuration data with additional SMA compact configuration data that were obtained as part of another project reported by Wang et al. (2014).

We used the Herschel Space Observatory images obtained from the Herschel Infrared Galactic Plane Survey (Hi-GAL) survey (Molinari et al. 2016) at $70 \mu \mathrm{m}$ with the Photoconductor Array Camera and Spectrometer (PACS) instrument. The standard pipeline data (level 2.5) were downloaded from the NASA/IPAC Infrared science archive ${ }^{2}$.

\section{Large- to small-scale properties of the massive "starless" cores}

The two clumps we targeted with SMA are embedded in two of the most prominent IRDCs. Figure 1 shows the large-scale, three-color view of the two IRDCs as seen with Spitzer (Churchwell et al. 2009; Carey et al. 2009). It shows the extended filamentary extinction features (Butler \& Tan 2009;

\footnotetext{
1 The Submillimeter Array is a joint project between the Smithsonian Astrophysical Observatory and the Academia Sinica Institute of Astronomy and Astrophysics, and is funded by the Smithsonian Institution and the Academia Sinica.

2 http://irsa.ipac.caltech.edu/applications/Herschel/
}

Kainulainen et al. 2013) and the main star formation sites embedded within them. Herschel $(70 \mu \mathrm{m})$ and APEX Large APEX BOlometer CAmera (LABOCA, $870 \mu \mathrm{m}$, Schuller et al. 2009) images of the two sources are also presented in Fig. 1.

The brightest $\mathrm{mm}$ source (source P1 in Carey et al. 2000, see Fig. 1) within the northern part of the IRDC G11.11-0.12 is a high-mass protostar (Pillai et al. 2006a; Gómez et al. 2011; Henning et al. 2010; Wang et al. 2014), while the southern mm clump we are interested in appears largely quiescent. The focus of this study is this secondary dust emission source (encircled region in the left panel of Fig. 1). It is clearly associated with a compact absorption feature at 70 and even at $100 \mu \mathrm{m}$ (see Fig. B.9 of Ragan et al. 2012). We name this source G10.99-0.08. No point sources are observed in the near or mid-infrared with either GLIMPSE (Churchwell et al. 2009) or MIPS Galactic Plane Survey (MIPSGAL; Carey et al. 2009) as well. The nearest protostellar candidate reported in this region is a $25 L_{\odot}\left(5 M_{\odot}\right)$ protostar outside our SMA field of view (FOV) and at a distance of $\sim 34$ arcsec from our central pointing (Henning et al. 2010). Single dish $\mathrm{NH}_{3}$ observations have shown that this region is unusually cold $(T \sim 12 \mathrm{~K})$ with a peak $\mathrm{H}_{2}$ column density of $2 \times 10^{22} \mathrm{~cm}^{-2}$ and total mass of $\sim 1580 M_{\odot}$ (Pillai et al. 2006b; Urquhart et al. 2015). No high-resolution observations have been discussed in the literature to date.

The IRDC G28.34+0.06 is more complex, with the brightest mm clump in IRDC G28.34+0.06 hosting a prominent IRAS source 18402-0403 (source P2 in Carey et al. 2000), while the next bright mm clump (source P1 following Carey et al. 2000, Fig. 1) hosts a young protocluster driving multiple collimated outflows (Wang et al. 2011; Zhang et al. 2009, 2015). Owing to its large mass reservoir and lack of obvious star formation indicators at IR wavelengths, the less prominent mm clump we are interested in (encircled region in the right panel of Fig. 1) has been studied in detail recently as a potential high-mass starless clump candidate (Tan et al. 2013; Kong et al. 2018). We name this source G28.33+0.07. Similar to G10.99-0.08, no point source has been reported in the near or mid-infrared with either GLIMPSE (Churchwell et al. 2009) or MIPSGAL (Carey et al. 2009). G28.33+0.07 is also dark at 70 and even at $100 \mu \mathrm{m}$ (see Fig. B.22 of Ragan et al. 2012). More recently, bipolar outflow emission towards the most massive core has been revealed in G28.33+0.07 (Feng et al. 2016; Tan et al. 2016; Kong et al. 2018).

Both observations and physical models of protostellar emission predict a direct correlation between $70 \mu \mathrm{m}$ flux and the internal luminosity of the source (Dunham et al. 2008). Tan et al. (2013) show that absorption at $100 \mu \mathrm{m}$ arises from very cold $(<12 \mathrm{~K})$ dust and is indicative of negligible internal heating. We analyzed the spectral energy distribution (SED) using all Herschel PACS and Spectral and Photometric Imaging Receiver (SPIRE) bands and LABOCA data. A detailed explanation of the SED fitting is given by König et al. (2017). SEDs for G10.99-0.08 and G28.33+0.07 are shown in Fig. A.1. The best-fit SED provides a good match to the observed flux at all wavelengths within the flux uncertainty. It corresponds to a bolometric luminosity (temperature) of $10 L_{\odot}$ $(9 \mathrm{~K})$ and $\sim 24 L_{\odot}(11 \mathrm{~K})$ for G10.99-0.08 and G28.33+0.07, respectively. These modest luminosities do not imply the presence of embedded sources: it is merely the luminosity generated by dust emission from a large mass of cold gas, as also indicated by the low dust temperatures derived as part of the gray-body fit. We computed the gas mass for the abovementioned temperature adopting a dust opacity at $870 \mu \mathrm{m}$ of $0.021 \mathrm{~cm}^{2} \mathrm{~g}^{-1}$ for thin ice mantles that have coagulated at a 

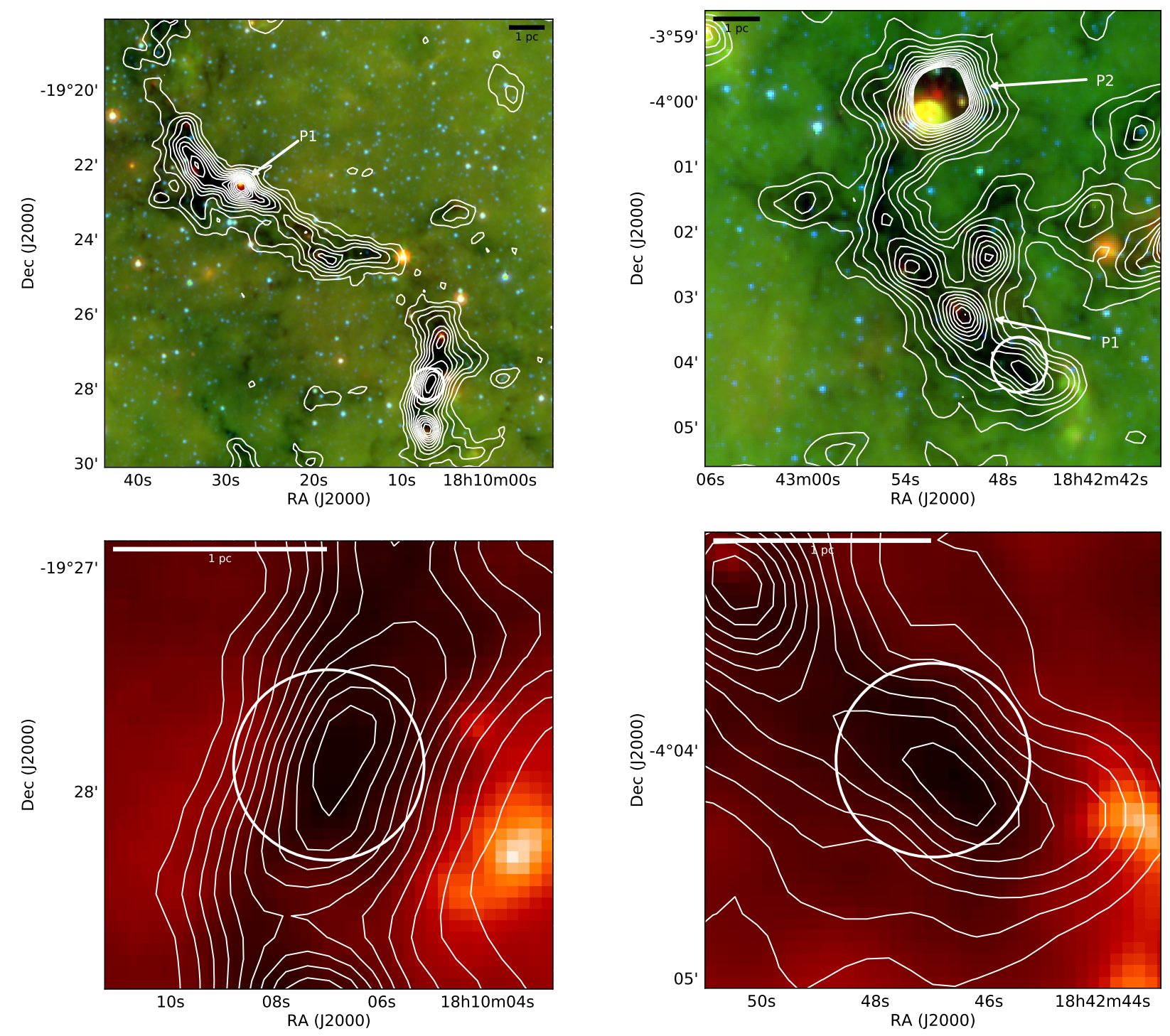

Fig. 1. Top panels: large-scale, three-color view of the IRDCs G11.11-0.12 (left panel) and G28.34+0.06 (right panel) obtained with Spitzer IRAC and MIPS data with $3.6 \mu \mathrm{m}$ (blue), $8.0 \mu \mathrm{m}$ (green), $24.0 \mu \mathrm{m}$ (red) from the GLIMPSE and MIPSGAL survey (Churchwell et al. 2009 ; Carey et al. 2009). Bottom panels: Herschel PACS $70 \mu$ m emission towards our targets G10.99-0.08 (left panel) and G28.33+0.07 (right panel; Molinari et al. 2016). The contour levels correspond to ATLASGAL $870 \mu \mathrm{m}$ emission (0.1-1.3 in steps of $0.1 \mathrm{mJy}$ beam $^{-1}$; Schuller et al. 2009). The starless core regions mapped with the SMA are shown as circles.

density of $10^{6} \mathrm{~cm}^{-3}$ for $10^{5} \mathrm{yr}$ (Ossenkopf \& Henning 1994). The total mass measured within the SMA field of view (FOV) of $51^{\prime \prime}$ with single dish (APEX) is 1183 and $960 M_{\odot}$ for G10.99-0.08 and G28.33+0.07, respectively. For a radius of $0.44 \mathrm{pc}(\mathrm{G} 10.99-0.08)$ and $0.59 \mathrm{pc}(\mathrm{G} 28.33+0.07)$, these values exceed the empirical mass-size threshold for high-mass star formation by a factor $>3$ (Kauffmann \& Pillai 2010) $)^{3}$. Thus, the SEDs and submillimeter emission for both sources are consistent with a cold, massive phase with little evidence of ongoing high-mass star formation.

We also used the Spitzer and Herschel non-detections to constrain the luminosity of the possible population of embedded protostars. The protostellar population is both heavily embedded (for the brightest continuum cores) and/or of low luminosity (distributed population of cores), below the detection limit of infrared observations up to $24 \mu \mathrm{m}$ flux. We can, however, use the

3 As prescribed in Kauffmann \& Pillai (2010), we scale down the submillimeter opacity by a factor 1.5 when computing the mass-size excess. information on $70 \mu \mathrm{m}$ flux density to constrain the protostellar luminosity of our targets. Dust radiative models of protostellar emission as well as empirical results from low-mass protostars have shown a strong correlation between $70 \mu \mathrm{m}$ flux and internal luminosity of a protostar. In particular, Dunham et al. (2008) show that the internal luminosity of a dense core is related to its flux density at $70 \mu \mathrm{m}$ wavelength, $S_{v}(70 \mu \mathrm{m})$, by (reformulated here for a distance "d" in $\mathrm{kpc}$ )

$L_{\text {int }}=2.4 L_{\odot} \cdot\left(\frac{S_{v}(70 \mu \mathrm{m})}{\mathrm{Jy}}\right)^{0.94} \cdot\left(\frac{d}{\mathrm{kpc}}\right)^{1.88}$,

where the numerical constants follow from Eq. (2) of Dunham et al. (2008). We use this relation ${ }^{4}$ to place an upper

4 Alternatively, one could also base the calculation on Table 2 of Dunham et al. (2008), which is based on a sparse sample of observational data. In that case, $L_{\text {int }}=2.2 L_{\odot} \cdot\left(S_{\nu}[70 \mu \mathrm{m}] / \mathrm{Jy}\right)^{0.94} \cdot(d / \mathrm{kpc})^{1.89}$ at $70 \mu \mathrm{m}$ wavelength, and $L_{\mathrm{int}}=25 L_{\odot} \cdot\left(S_{v}[24 \mu \mathrm{m}] / \mathrm{Jy}\right)^{0.72} \cdot(d / \mathrm{kpc})^{1.45}$ at $24 \mu \mathrm{m}$ wavelength. 
limit on the total internal luminosity of protostars in our targets. Since our sources are dark at $70 \mu \mathrm{m}$, we use the $90 \%$ point-source completeness limit $\sim 0.5 \mathrm{Jy}$ for the $70 \mu \mathrm{m}$ emission reported by the Herschel infrared Galactic Plane Survey, Hi-GAL (Fig. 9 of Molinari et al. 2016). This provides us with a luminosity upper limit of 14 (24) $L_{\odot}$ for G10.99-0.08 $(\mathrm{G} 28.33+0.07)$. This is the predicted protostellar luminosity resulting from internal heating and comparable to that derived from our integrated SED over all wavelengths mentioned above.

At higher spatial resolution with the SMA, the single dish submillimeter emission in both sources breaks up into a string of roughly equidistant compact continuum peaks. We detect compact continuum emission at the position of the $70 \mu \mathrm{m}$ absorption feature for both targets (see Fig. 2). We identified sub-structures by performing a dendrogram analysis (Rosolowsky et al. 2008) on this dataset to segment the dust continuum emission. The segmentation starts at a threshold $\geq 5 \sigma$ noise level in steps of $1 \sigma$ and yields several cores within the single dish peak. Adopting a dust opacity at $230 \mathrm{GHz}$ of $0.01 \mathrm{~cm}^{2} \mathrm{~g}^{-1}$ (Ossenkopf \& Henning 1994) and a dust temperature of $15 \mathrm{~K}$, we also derive total gas mass within the dendrogram structures. Our $3 \sigma$ detection threshold corresponds to a mass sensitivity of $\sim 1$ and $\sim 2 M_{\odot}$ beam $^{-1}$ for G10.99-0.08 and G28.33+0.07, respectively. The mass range of $1.3-33 M_{\odot}$ covers a large range from solarmass to high-mass cores (see Table B.2). The brightest dust continuum cores (we will refer to it as "core 1" in both targets) in both sources exceed $10 M_{\odot}$.

The structure of our continuum emission for G28.33+0.07 is consistent with the published ALMA observations of Tan et al. (2013). Based on ALMA $\mathrm{N}_{2} \mathrm{D}^{+}$(3-2) integrated intensity and dust continuum emission, Tan et al. (2013) identify two starless core candidates $\mathrm{C} 1-\mathrm{N}$ and $\mathrm{C} 1-\mathrm{S}$ at 2.3 arcsec resolution. Their follow-up ALMA observations at approximately two times better angular resolution reveal that $\mathrm{C} 1-\mathrm{S}$ core fragments into C1-Sa and C1-Sb (Tan et al. 2016). Two more dust continuum cores, $\mathrm{C} 1 \mathrm{a}$ and $\mathrm{C} 1 \mathrm{~b}$, located away from $\mathrm{C} 1-\mathrm{S}$ and $\mathrm{C} 1-\mathrm{N}$ cores are identified in this data (see Fig. 2). All four cores are found to drive outflows and are thus considered protostellar in nature. Using the mm fluxes given in their Table 1 and using the same dust opacity and temperature mentioned above for our SMA observations, we estimate that these cores have masses of $\sim 13$ (C1-Sa), 1.8 (C1-Sb), $2.3(\mathrm{C} 1 \mathrm{a})$, and $2.3(\mathrm{C} 1 \mathrm{~b}) M_{\odot}$. While our SMA image is centered roughly on $\mathrm{C} 1-\mathrm{N}$, the Tan et al. ALMA pointing center is closer to $\mathrm{C} 1-\mathrm{S}$ and the SMA $1.3 \mathrm{~mm}$ primary beam is twice as large as the ALMA primary beam. Therefore, beyond $\mathrm{C} 1-\mathrm{N}$ towards the north, we should not expect any overlap in structures. Within the region of overlap, the position of $\mathrm{C} 1-\mathrm{N}$ and $\mathrm{C} 1-\mathrm{S}$ coincides well with our observations and $\mathrm{C} 1-\mathrm{N}$ remains unfragmented even at the higher resolution of ALMA. While we detect some extended emission towards C1-a and $\mathrm{C} 1-\mathrm{b}$, at the resolution and sensitivity, our dendrogram analysis does not pick these up as distinct cores.

\section{Outflow search}

Our primary goal is to understand the starless versus protostellar nature of the cores in these apparently "starless" regions. Since molecular outflows are one of the most unambiguous tracers of protostellar emission, we search for high-velocity CO 2-1 emission in our high-resolution SMA data. The outflow emission would be traced in the high velocity wings of $\mathrm{CO}$.

We used our previously published $\mathrm{NH}_{3}$ (Pillai et al. 2006b) data to confine the extent of dense gas emission at the systemic velocity. The systemic velocities are $29.2 \pm 3$ and $78.4 \pm 3 \mathrm{~km} \mathrm{~s}^{-1}$ for G10.99-0.08 and G28.33+0.07, respectively. Outside of this range, we define the high-velocity emission from $\mathrm{CO}$. However, our data reveal the presence of complex extended $\mathrm{CO}$ emission in this high-velocity range. This emission is uncorrelated with the cloud structure and has velocities as high as $\sim \pm 50 \mathrm{~km} \mathrm{~s}^{-1}$ around the systemic velocity. This is exemplified in the integrated spectrum obtained on the brightest continuum sources (see Fig B.1). We note that the negative spectral features in Fig. B.1 arise from missing short spacing information from diffuse emission in an interferometer image. A significant fraction of emission features do not show any clear collimated structure that could be associated with an outflow component. The presence of such complex emission is not unexpected given that the two clouds are located well within the inner Galactic Plane $|b|<0.2$ degrees and therefore could be potentially contaminated by unrelated interstellar medium (ISM) features along their line of sight. A wide range in velocities between -10 and $+50 \mathrm{~km} \mathrm{~s}^{-1}$ would be consistent with galactic rotation in the direction of both clouds (see Fig. 3, Dame et al. 2001) and can contaminate ${ }^{12} \mathrm{CO}$ data from unrelated ISM features along the cloud line of sight. Such ISM structures may be dense $\left(\rho>10^{4} \mathrm{~cm}^{-3}\right)$ or diffuse. Dense structures, however, would also be detected in dense gas tracers such as $\mathrm{NH}_{3}$. There is no indication for such a component outside of the systemic velocity range discussed above. Diffuse emission along the line of sight with little sub-structure would be mostly filtered out by the interferometer. However, the SMA in its subcompact configuration is sensitive to structures as extended as $\sim 0.5-0.7 \mathrm{pc}$ at $3.6-4.8 \mathrm{kpc}$ (for a minimum baseline length of $\sim 9.5 \mathrm{~m})$.

In order to extract only the outflow related component, we do the following. First, we use a 1 dimensional non-LTE line radiative transfer model RADEX (van der Tak et al. 2007) to calculate the expected brightness temperature of a diffuse $\left(\geq 1 A_{V}\right)$ cloud component along the line of sight. Using the standard ${ }^{12} \mathrm{C} /{ }^{13} \mathrm{C}$ ratio $(77)$ and ${ }^{12} \mathrm{CO}$ abundance $\left(2 \times 10^{-4}\right)$, density of $1000 \mathrm{~cm}^{-3}$. and temperature of $15 \mathrm{~K}$, we derive a brightness temperature $>0.5 \mathrm{~K}$. We extract $5 \times 5 \operatorname{arcmin}^{2}$ maps of ${ }^{13} \mathrm{CO} 2-1$ map from APEX (Schuller et al. 2017) and 1-0 map from Five College Radio Astronomical Observatory (FCRAO; Jackson et al. 2006) for G10.99-0.08 and G28.33+0.07, respectively. We then identify velocities in the channel maps associated with all structures extended with respect to the beam above the $0.5 \mathrm{~K}$ threshold. These velocity ranges are explicitly excluded when creating moment maps in the ${ }^{12}$ CO SMA data. Due to the expanding motions, outflows would exhibit sharp velocity gradients when visualized in the position-position-velocity (PPV) space, while intervening clouds would have a sheet-like distribution. Therefore, as a final check, we use a 3 dimensional volume rendering data visualization software package Glue ${ }^{5}$ to explore structures in PPV space and only consider structures associated with outflows. Additionally, for the two clouds, we also flag out the shortest baselines $(<10 \mathrm{k} \lambda$ or $13 \mathrm{~m})$ to further filter out large-scale structures. A $u v$ distance of $10 k \lambda$ is merely the best compromise that we found between retaining outflow emission while removing large-scale features and does not have any physical significance. We note that this process would also remove some outflow emission (and thus underestimate outflow properties). Due to the nearer distance (sensitive to more extended emission for a given beam) and more severe line of sight contamination, we apply this filtering for the whole velocity range for G10.99-0.08. For G28.33+0.07 only the velocity range

\footnotetext{
http://glueviz.org
} 
$84-88 \mathrm{~km} \mathrm{~s}^{-1}$ (more than $6 \mathrm{~km} \mathrm{~s}^{-1}$ red-shifted from the cloud local standard of rest (LSR) velocity) is subjected to the filtering constraint since there is significant emission from outflows as well as ambient gas only in this range. The resulting ${ }^{12} \mathrm{CO}$ structures are shown in the right panels of Fig. 2. This emission is complex and not always bipolar presumably because the outflows we detect at our resolution are unresolved and likely to be a combination of outflows from unresolved cluster members. Therefore, defining specific outflow features has limited meaning and we shall revisit this with high-resolution, high-sensitivity ALMA data (Sanhueza et al., in prep.).

Having made an extensive effort to exclude emission unassociated with the cloud, we conclude that the high-velocity emission shown in Fig. 2 is very likely associated with outflows. Thus, we have our final maps that allow us to derive combined outflow properties of the system such as mass, momentum, and energy. We do not calculate dynamical timescales (and therefore mechanical force, momentum, and energy injection rates) for these outflows. Derived outflow properties are listed in Table B.1. It is important to note that we ignore velocities close to the ambient velocity, adopt an $\mathrm{CO}$ excitation temperature at the lower end of the usual range of $10-50 \mathrm{~K}$, assume that ${ }^{12} \mathrm{CO}$ is optically thin, and we do not account for the (unknown) inclination angle of the outflow and spatial filtering of extended emission in our observations. When accurately constrained and considered in the mass calculation, all these effects conspire to significantly increase the outflow mass by an order of magnitude or more (Dunham et al. 2014). Therefore, our outflow parameters are strictly very conservative lower limits. In fact, deeper ALMA observations of the most massive outflows in G28.33+0.07 (Tan et al. 2016) find outflow parameters that are an order of magnitude higher than our values. For G28.33+0.07 we compared in detail our SMA outflow detections (our Fig. 2, bottom right panel) with that of the higher resolution outflows detected in Tan et al. (their Fig. 1, bottom right panel). It is evident from this comparison that the main outflow emission in our SMA data towards the unresolved source C1-S is actually two outflows driven by $\mathrm{C} 1-\mathrm{Sa}$ and $\mathrm{C} 1-\mathrm{Sb}$. Tan et al. find the outflow masses $\left(\sim 1 M_{\odot}\right)$ and momenta $\left(\sim 11 M_{\odot} \mathrm{km} \mathrm{s}^{-1}\right)$ from $\mathrm{C} 1-\mathrm{Sa}$ and $\mathrm{C} 1-\mathrm{Sb}$ to be similar within the expected errors. In their more recent work reporting even higher resolution observations (0.2"), Kong et al. (2018) report that the CO outflow momentum flux of the lower mass source $\mathrm{C} 1-\mathrm{Sb}$ is only moderately smaller and has a larger outflow cavity opening angle than that of $\mathrm{C} 1-\mathrm{Sa}$, suggesting that $\mathrm{C} 1-\mathrm{Sb}$ is a low- or intermediatemass protostar at a more advanced evolutionary phase. Several red and blue-shifted features outside of these two outflows appear in their map. The lack of resolution makes it difficult to be certain, however these would roughly overlap with our weak outflow features towards $\mathrm{C} 1-\mathrm{N}, \mathrm{C} 1-\mathrm{a}$, and $\mathrm{C} 1-\mathrm{b}$. The nature (such as mass or momentum) of these outflows are not discussed in Tan et al. or Kong et al. The majority of our brighter outflow features are located outside their FOV. At a similar sensitivity level, collimated outflows have been detected with the SMA towards IR bright protostars in the two IRDCs harboring G10.99-0.08 and G28.33+0.07 (Wang et al. 2011, 2014). These outflows have outflow masses and momenta much higher on average than those towards the IR quiet regions that we are studying in the same cloud. These differences are most likely due to the differences in their evolutionary stages as also evidenced by the different IR luminosity. We note that outflow properties are similar to those reported for low-mass protostars in a more evolved high-mass protocluster by Cyganowski et al. (2017).

\section{Discussion}

We detect several outflows within $\sim 1$ arcmin diameter of the two targets. This is intriguing given the lack of infrared emission within the whole clump.

\subsection{Nature of the $24 / 70 \mu m$ dark massive core: high-mass star progenitors}

In both targets, the brightest continuum core of $>10 M_{\odot}$ is associated with at least one outflow. In G28.33+0.07, highresolution ALMA observations have confirmed two distinct outflows. The outflows newly detected in G10.99-0.08 by us validate that both massive apparently starless cores are in fact not starless. At $0.005 \mathrm{pc}$ resolution, the most massive core in the $\mathrm{G} 28.33+0.07$ region has $30 M_{\odot}$ (Kong et al. 2018). At a roughly similar spatial resolution $(0.007 \mathrm{pc})$, the most massive core in nearby low-mass clusters like NGC 1333 has a mass of $2 M_{\odot}$ (Plunkett et al. 2015). Similarly, ALMA higher-resolution data for G10.99-0.08 suggest that the main core (core 1) shows little fragmentation as also suggested by its compactness at SMA resolution (Sanhueza et al., in prep.). The outflow wings of the most massive core in both sources extend to $>50 \mathrm{~km} \mathrm{~s}^{-1}$ (this work, Sanhueza et al., in prep., Kong et al. 2018), which is typical of high-mass outflows (Maud et al. 2015). These outflows are also very compact with respect to the SMA beam, implying a highly collimated powerful outflow. It is clear from the core mass, compactness of the outflow, and its extent to very high velocities $\left(>50 \mathrm{~km} \mathrm{~s}^{-1}\right)$ as seen towards massive outflows that the most massive cores (core 1) in G10.99-0.08 and G28.33+0.07 stand out in their outflow properties from the rest of the outflow features. These data in summary suggest that high-mass stars in an early state of evolution are the drivers of these outflows. However, can a protostar with a massive envelope exhibit such a low luminosity of only $10-20 L_{\odot}$ and still build up over time to a massive star?

To address this question, we compare the observed envelope properties such as mass and bolometric luminosity to that predicted by radiative transfer (RT) models focused on massive young stellar objects (YSOs). A large RT model grid has been developed for SED of massive YSOs by Molinari et al. (2008). Different models have been explored for five different final stellar masses from 6.5 to $35 M_{\odot}$. Comparing our observations to their model predictions, the observed low bolometric luminosity of 10-20 $L_{\odot}$ is to be expected for massive cores in the first 50000 years of evolution. Models with final stellar masses of $6.5,8$, and $13.5 M_{\odot}$ reach such low luminosities within a short time of $<50000$ years (see their Fig. 9 and Table 7). According to Molinari et al. (2008), these objects would be the Class 0 analog in the classical classification established for low-mass YSOs. The duration of the phase consistent with our observations rapidly decreases with increasing stellar mass. It thus seems plausible that the actual duration of the low-luminosity phase observed by us is well below the upper limit of $50000 \mathrm{yr}$. Models of more massive stars $\left(18 M_{\odot}\right.$ and higher) are not consistent with our data.

We also compare our observations with radiative transfer models based on the turbulent core theory of massive star formation (McKee \& Tan 2003). These models include protostellar evolution in a self-consistent matter (Zhang et al. 2014; Zhang \& Tan 2018). Depending on the initial mass of the turbulent core, $\left(M_{\mathrm{c}}\right)$, and mean clump mass surface density, $\Sigma_{\mathrm{cl}}$, the final stellar masses in their model are in the range $20-90 M_{\odot}$. Figure 6 in Zhang et al. (2014) follows the evolution of massive 

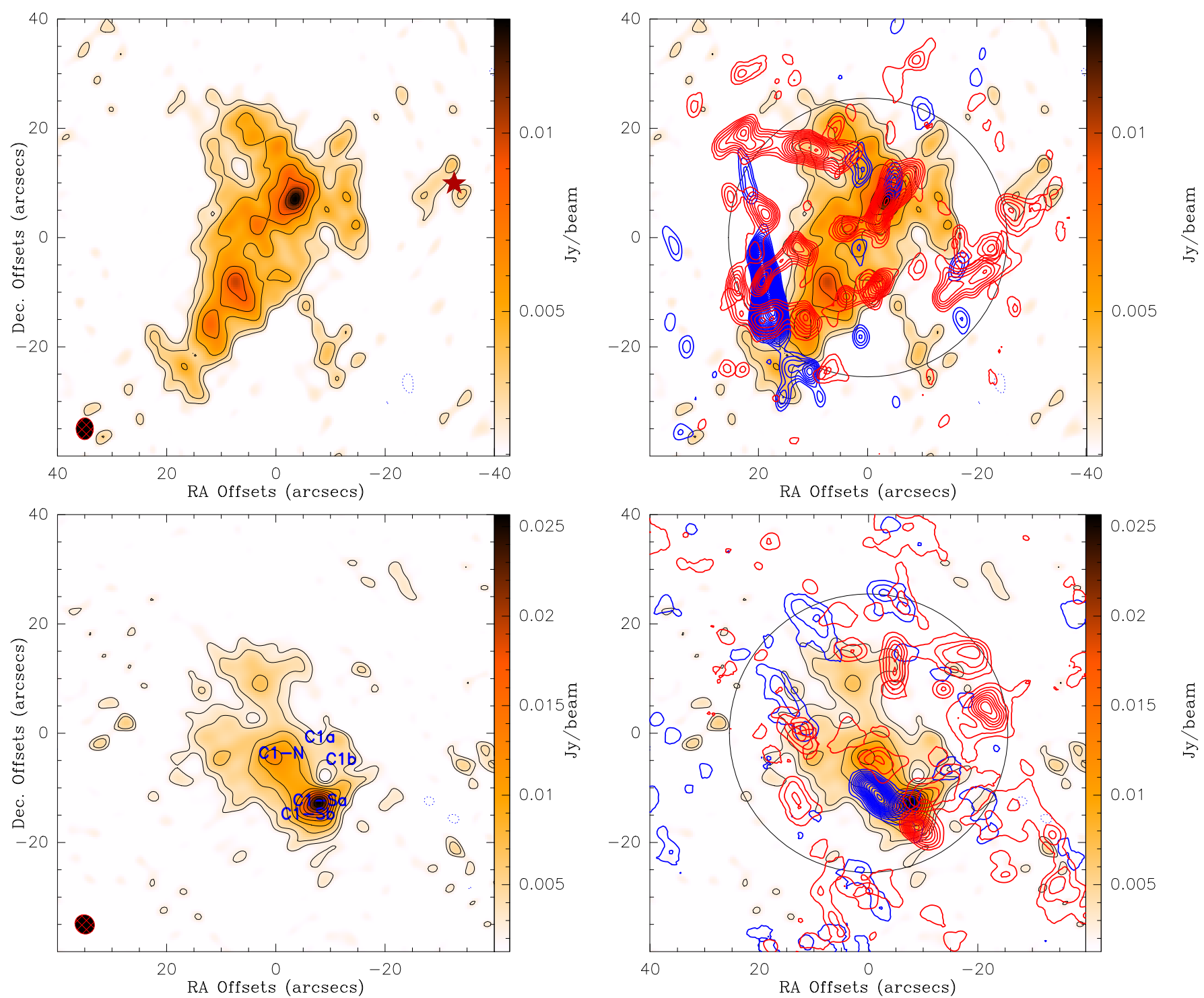

Fig. 2. Left panels: SMA $230 \mathrm{GHz}$ thermal dust continuum emission towards G10.99-0.08 (top panel) and G28.33+0.07 (bottom panel). The levels start at $2 \sigma, 3 \sigma$, in steps of $2 \sigma$. Right panels: SMA CO 2-1 integrated intensity emission at high velocities as contours overlaid on SMA dust continuum observations towards G10.99-0.08 (top panel) and G28.33+0.07 (bottom panel). The contours start at $6 \sigma$, in steps of $2 \sigma$. The circle shows the SMA FOV at $230 \mathrm{GHz}$. The ellipse in the lower left corner represents the SMA synthesized beam size. The magenta star in the top left panel for G10.99-0.08 corresponds to a Herschel identified low-mass protostar outside the SMA FOV by Henning et al. (2010).

protostellar cores with different $\Sigma_{\mathrm{cl}}$. For $\Sigma_{\mathrm{cl}}=0.316 \mathrm{~g} \mathrm{~cm}^{-2}$, which is roughly representative of our clumps, progenitors of massive protostars have luminosities as low as our observed luminosities in the first 20000 years of evolution. We thus conclude that the embedded stars of low luminosity in our target regions are consistent with being young, high-mass stars. It is impossible to determine the evolutionary status of these sources with absolute certainty. Still, the combination of core mass, strong outflow, and modest limits to the luminosity make young, high-mass stars the most plausible candidates for these sources.

The predicted model luminosities and therefore ages from both these models, however, might be significantly offset from the actual observed luminosities. Known as the "protostellar luminosity problem", the observed protostellar luminosities of low-mass protostars are approximately ten times less luminous than expected from theories of protostellar evolution (Kenyon et al. 1990; Evans et al. 2009). Out of the many solutions proposed to explain this discrepancy, the most favored is that of an episodic accretion, where an accretion is extremely variable and the bulk of the protostellar mass is accreted in rare bursts of high accretion. Recently, observational evidence for such accretion events has also been building up for massive stars (Caratti O Garatti et al. 2017; Hunter et al. 2018). If such accretion events are indeed the main source of accretion, then the model luminosities discussed above need to be refined by an order of magnitude or higher. This would imply that realistic luminosities from models should be lower and the protostellar lifetimes longer than those predicted by the current models.

The large-scale mass reservoir $\left(\sim 1000 M_{\odot}\right.$ in both targets) would allow the formation of massive protostars in both sources (see, for example, Sanhueza et al. 2017). For G28.33+0.07, higher resolution ALMA observations reveal a protostellar envelope towards the central object of between 20 and $50 M_{\odot}$ (Kong et al. 2018). Additionally, SiO 2-1 and CO 2-1 outflow properties discussed in Feng et al. (2016) and Tan et al. (2016) respectively, support the scenario that the central object is a 
massive protostellar core that is in a relatively early phase of collapse. Based on the models discussed above that are relevant to high-mass protostellar evolution, we conclude that for final stellar masses between 6.5 and $20 M_{\odot}$, we can indeed reproduce the observed low luminosity and protostellar masses for G10.99-0.08 and G28.33+0.07 as being associated to YSOs at the earliest stage in the evolution of massive protostars.

\subsection{Nature of other outflow driving sources without $\mathrm{mm}$ continuum}

What we see in the larger environment of the clump away from the main continuum core is a complex superposition of outflows. Since these outflows are not associated with any continuum cores, it is important to compare the combined structure of these outflows with nearby, resolved, better understood templates of overlapping outflows. We use the nearby, resolved young cluster region in NGC 1333 as our outflow template. We chose this particular region because the spatial resolution of these observations match the spatial resolution of our SMA G10.99-0.08 observations $(\sim 0.06 \mathrm{pc}$ at $3.6 \mathrm{kpc})$. Clearly our region hosts more massive cores than NGC 1333, however it also appears to harbor a small, distributed (over $\sim 1 \mathrm{pc}$ ) population of young, low-mass YSOs at the same time.

We note that for $\mathrm{G} 28.33+0.07$, in the region where there is spatial overlap between our SMA data and ALMA data in Tan et al. (2016), Tan et al. report two low-mass dust continuum cores of $\sim 2 M_{\odot}$ named C1a and C1b (see Sect. 3 ) that drive outflows and are thus considered low- or intermediate-mass protostars. Most of our outflows are outside this region of overlap for $\mathrm{G} 28.33+0.07$.

First we investigate the effect of a limited resolution on the $\mathrm{CO}$ observations. We used a published CO 1-0 outflow map (Arce et al. 2010) towards the NGC 1333 star-forming region within the Perseus molecular cloud. Observations have shown that the NGC 1333 region is forming low- and intermediatemass stars and is the most active site of star formation in Perseus (Bally et al. 2008). We selected an active sub-region "Area II" following the naming convention in Arce et al. (see their Fig. 6). Arce et al. detect several outflows in the region and report the extent of outflow emission in blue and red lobes. We downloaded their archived FITS data cubes and generated integrated intensity map for the average range identified in their work (see their Fig. 4). Adopting the known distances to Perseus $(250 \mathrm{pc})$ and G10.99-0.08 (3.6 kpc), we scaled the integrated intensity image to the distance of the G10.99-0.08 region. We then extracted a 1 arcmin region (roughly matching the SMA field of view of $\sim 51$ arcsec) around the scaled image and performed a visual comparison of the outflow properties. As shown in Fig. B.3, five outflows from Arce et al. (Flows 1-5) have been identified in red-shifted emission within the same spatial scale as our SMA data. The total mass within all outflows is $3.5 M_{\odot}$ while we can only place a lower limit of $\sim 0.05 M_{\odot}$ for the outflow mass in G10.99-0.08. We have not taken into account the effect of interferometer spatial filtering that would filter out the large-scale emission of these outflows and would make them appear more compact and less massive. The number of outflow features, and the general overall morphology of the features identified as potential outflows in our data is therefore broadly consistent with what one might expect based on the resolved emission from a low-mass cluster-forming region like NGC 1333

The lack of thermal dust continuum cores is to be understood as well. Our dust continuum observation is sensitive to $\sim 1-2 M_{\odot}$ core. The sources in NGC 1333, however, have sub-solar masses
(Plunkett et al. 2013, except for IRAS4A), significantly below our detection limit. In the mid-Infrared (mid-IR), Dunham et al. (2015) report 125 protostars based on MIPS $24 \mu \mathrm{m}$ band observations. We used the Spitzer MIPSGAL Legacy Program that imaged the $24 \mu \mathrm{m}$ inner galactic plane (Carey et al. 2009) to search for mid-IR counterparts driving outflows in G10.99-0.08 and G28.33+0.07. Specifically, we used the $24 \mu \mathrm{m}$ point source catalog based on MIPSGAL (Gutermuth \& Heyer 2015) to identify all protostars within the SMA FOV. This search yields only a single point source in G10.99-0.08 and none in G28.33+0.07. The location of the $24 \mu \mathrm{m}$ point source in G10.99-0.08 is distinctly offset from all dust continuum peaks along the main ridge. In order to understand the effect of distance, we scaled the Dunham et al. point source flux densities to those at a distance of $3.6 \mathrm{kpc}$ and set a sensitivity cutoff at $1 \mathrm{mJy}$ similar to that used by Gutermuth \& Heyer (2015). We can then predict that 21 YSOs in NGC 1333 are expected to be detected at $3.6 \mathrm{kpc}$. However, the extinction maps towards G10.99-0.08 and G28.33+0.07 (Kainulainen et al. 2013; Butler \& Tan 2009) reveal that deep within the galactic plane, even the larger scale environment of the IRDCs that we are probing experiences high extinction. A $30 \mathrm{mag}$ visual extinction can easily diminish the flux densities at $24 \mu \mathrm{m}$ by a factor of five (Flaherty et al. 2007), resulting in an equivalent detection threshold of $5 \mathrm{mJy}$. This would reveal at most approximately four protostars at $3.6 \mathrm{kpc}$ in NGC 1333, consistent with our finding of a single point source in the catalog above a cutoff of $1 \mathrm{mJy}$. Therefore, we conclude that the observed complex outflow emission, as well as the lack of continuum cores and MIR sources associated with these outflows are consistent with a distributed population of low-mass protostars.

\subsection{Cluster formation in IRDCs}

An initial search for low-mass star formation towards a distant high-mass star-forming IRDC revealed a distributed population of low-mass pre-main sequence stars (Foster et al. 2014). These were near-IR ( $K$ band) observations that could only detect low-mass protostars forming in tghe inter-clump medium of the IRDC and not towards the high column density clumps where massive protostars are embedded. A massive more evolved (IRbright) clump located within the same IRDC as G28.33+0.07 was followed up with high sensitivity ALMA observations (Zhang et al. 2015). These observations together with a careful treatment of interferometer effects did not find any evidence for a distributed population of low-mass sources either in the form of low-mass cores or outflows close to the high-mass cluster. This led Zhang et al. to conclude that low-mass stars would form later, while they also found that lower mass cores are forming within the immediate vicinity of higher mass objects. In contrast to their results, a recent ALMA observation of a more evolved massive star-forming region have found evidence for a population of low-mass cores in the outer boundaries $(\sim 0.17 \mathrm{pc}$ median value) of the main mass reservoir (Cyganowski et al. 2017). The largest spatial scales probed might play a role in the interpretation of low-mass protostars. Cyganowski et al. observations were made in mosaic mode covering a spatial extent of $0.7 \mathrm{pc}$, while the Zhang et al. study is a single pointing study. Different initial conditions or differences in evolutionary phase might also play an important role. Our observations discussed here towards an even earlier phase of massive star formation than studies so far, show potential evidence for low-mass star formation distributed in a larger $(0.6 \mathrm{pc})$ environment around the accreting massive core. This is gathering evidence for a scenario where low-mass stars either form first or form coevally with high-mass stars. 


\section{Conclusion}

Clusters play a very important role in the formation of massive stars (Lada \& Lada 2003). However, we are yet to understand the detailed physics of this process. Our goal was to understand massive star and cluster formation at the earliest phase by studying two massive cold clumps seemingly with no evidence of star formation.

- We detect high-velocity emission in CO 2-1 towards two $100 \mu \mathrm{m}$ dark massive clumps that show no other evidence of star formation.

- The most massive cores in both sources are driving outflows. Their properties are consistent with the youngest phase $(<50000 \mathrm{yr})$ in evolution of a massive protostar.

- Our observations reveal several other potential outflow features distributed across a region of $0.6 \mathrm{pc}$ distinctly separated from the central massive cores.

- The lack of massive continuum cores and the combined outflow properties indicate that the driving sources of these sources are likely of low-mass (solar or sub-solar) origin.

- Our observations towards such a very early phase of massive star formation indicate that low-mass stars might form first or coevally with massive stars.

Acknowledgements. We thank the anonymous referee for a critical and constructive review of this manuscript. T.P. and K.W. acknowledge support from the Deutsche Forschungsgemeinschaft, DFG via the SPP (priority program) 1573 "Physics of the ISM". P.S. was financially supported by Grant-in-Aid for Scientific Research (KAKENHI Number 18H01259) of the Japan Society for the Promotion of Science (JSPS). This publication makes use of molecular line data from the Boston University-FCRAO Galactic Ring Survey (GRS). This research has made use of the VizieR catalog access tool, CDS, Strasbourg, France.

\section{References}

Arce, H. G., Borkin, M. A., Goodman, A. A., Pineda, J. E., \& Halle, M. W. 2010, ApJ, 715, 1170

Bally, J., Walawender, J., Johnstone, D., Kirk, H., \& Goodman, A. 2008, in Handbook of Star Forming Regions, ed. B. Reipurth, 1, 308

Bontemps, S., Motte, F., Csengeri, T., \& Schneider, N. 2010, A\&A, 524, A18

Butler, M. J., \& Tan, J. C. 2009, ApJ, 696, 484

Butler, M. J., \& Tan, J. C. 2012, ApJ, 754, 5

Caratti O Garatti, A., Stecklum, B., Garcia Lopez, R., et al. 2017, Nature Physics, 13,276

Carey, S. J., Feldman, P. A., Redman, R. O., et al. 2000, ApJ, 543, L157

Carey, S. J., Noriega-Crespo, A., Mizuno, D. R., et al. 2009, PASP, 121, 76

Chen, H., Liu, S., Su, Y., \& Zhang, Q. 2010, ApJ, 713, L50

Churchwell, E., Babler, B. L., Meade, M. R., et al. 2009, PASP, 121, 213
Cyganowski, C. J., Brogan, C. L., Hunter, T. R., et al. 2017, MNRAS, 468, 3694 Dame, T. M., Hartmann, D., \& Thaddeus, P. 2001, ApJ, 547, 792

Dunham, M. M., Crapsi, A., Evans, II., N. J., et al. 2008, ApJS, 179, 249

Dunham, M. M., Arce, H. G., Mardones, D., et al. 2014, ApJ, 783, 29

Dunham, M. M., Allen, L. E., Evans, II., N. J., et al. 2015, ApJS, 220, 11

Evans, N. J., Dunham, M. M., Jørgensen, J. K., et al. 2009, ApJS, 181, 321

Feng, S., Beuther, H., Zhang, Q., et al. 2016, A\&A, 592, A21

Flaherty, K. M., Pipher, J. L., Megeath, S. T., et al. 2007, ApJ, 663, 1069

Fontani, F., Palau, A., Caselli, P., et al. 2011, A\&A, 529, L7

Foster, J. B., Arce, H. G., Kassis, M., et al. 2014, ApJ, 791, 108

Gómez, L., Wyrowski, F., Pillai, T., Leurini, S., \& Menten, K. M. 2011, A\&A, 529, A161

Gutermuth, R. A., \& Heyer, M. 2015, AJ, 149, 64

Henning, T., Linz, H., Krause, O., et al. 2010, A\&A, 518, L95

Hunter, T. R., Brogan, C. L., MacLeod, G. C., et al. 2018, ApJ, 854, 170

Jackson, J. M., Rathborne, J. M., Shah, R. Y., et al. 2006, ApJS, 163, 145

Kainulainen, J., Ragan, S. E., Henning, T., \& Stutz, A. 2013, A\&A, 557, A120

Kauffmann, J., \& Pillai, T. 2010, ApJ, 723, L7

Kenyon, S. J., Hartmann, L. W., Strom, K. M., \& Strom, S. E. 1990, AJ, 99, 869

Kong, S., Tan, J. C., Caselli, P., et al. 2016, ApJ, 821, 94

Kong, S., Tan, J. C., Caselli, P., et al. 2018, ApJ, 867, 94

König, C., Urquhart, J. S., Csengeri, T., et al. 2017, A\&A, 599, A139

Lada, C. J., \& Lada, E. A. 2003, ARA\&A, 41, 57

Lu, X., Zhang, Q., Wang, K., \& Gu, Q. 2015, ApJ, 805, 171

Maud, L. T., Moore, T. J. T., Lumsden, S. L., et al. 2015, MNRAS, 453, 645

McKee, C. F., \& Tan, J. C. 2003, ApJ, 585, 850

Molinari, S., Pezzuto, S., Cesaroni, R., et al. 2008, A\&A, 481, 345

Molinari, S., Schisano, E., Elia, D., et al. 2016, A\&A, 591, A149

Ossenkopf, V., \& Henning, T. 1994, A\&A, 291, 943

Pillai, T., Wyrowski, F., Menten, K. M., \& Krügel, E. 2006a, A\&A, 447, 929

Pillai, T., Wyrowski, F., Carey, S. J., \& Menten, K. M. 2006b, A\&A, 450, 569

Pillai, T., Kauffmann, J., Wyrowski, F., et al. 2011, A\&A, 530, A118

Plunkett, A. L., Arce, H. G., Corder, S. A., et al. 2013, ApJ, 774, 22

Plunkett, A. L., Arce, H. G., Corder, S. A., et al. 2015, ApJ, 803, 22

Ragan, S., Henning, T., Krause, O., et al. 2012, A\&A, 547, A49

Rosolowsky, E. W., Pineda, J. E., Kauffmann, J., \& Goodman, A. A. 2008, ApJ, 679,1338

Sanhueza, P., Jackson, J. M., Zhang, Q., et al. 2017, ApJ, 841, 97

Schuller, F., Menten, K. M., Contreras, Y., et al. 2009, A\&A, 504, 415

Schuller, F., Csengeri, T., Urquhart, J. S., et al. 2017, A\&A, 601, A124

Tan, J. C. 2016, EAS Pub. Ser., 75, 105

Tan, J. C., Kong, S., Butler, M. J., Caselli, P., \& Fontani, F. 2013, ApJ, 779, 96

Tan, J. C., Kong, S., Zhang, Y., et al. 2016, ApJ, 821, L3

Urquhart, J. S., Figura, C. C., Moore, T. J. T., et al. 2015, MNRAS, 452, 4029

van der Tak, F. F. S., Black, J. H., Schöier, F. L., Jansen, D. J., \& van Dishoeck, E. F. 2007, A\&A, 468, 627

Wang, Y., Zhang, Q., Pillai, T., Wyrowski, F., \& Wu, Y. 2008, ApJ, 672, L33

Wang, K., Zhang, Q., Wu, Y., \& Zhang, H. 2011, ApJ, 735, 64

Wang, K., Zhang, Q., Testi, L., et al. 2014, MNRAS, 439, 3275

Wang, K., Testi, L., Burkert, A., et al. 2016, ApJS, 226, 20

Zhang, Q., Wang, Y., Pillai, T., \& Rathborne, J. 2009, ApJ, 696, 268

Zhang, Q., Wang, K., Lu, X., \& Jiménez-Serra, I. 2015, ApJ, 804, 141

Zhang, Y., \& Tan, J. C. 2018, ApJ, 853, 18

Zhang, Y., Tan, J. C., \& Hosokawa, T. 2014, ApJ, 788, 166 


\section{Appendix A: Dust continuum core properties and}

\section{noise-levels}
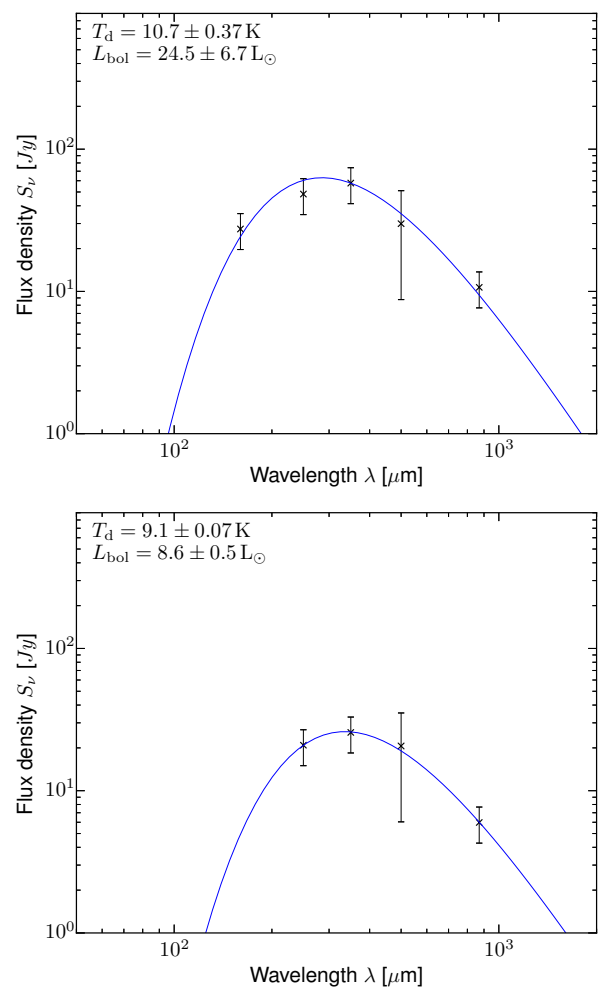

Fig. A.1. Spectral energy distribution based on Herschel (Molinari et al. 2016) and LABOCA data (Schuller et al. 2009) for G10.99-0.08 (top panel) and G28.33+0.07 (bottom panel). The data at different wavelengths are shown as squares with error bars. The temperature and bolometric luminosity obtained based on the SED fit assuming a dust opacity ( $\beta=1.8$ ) is also shown (König et al. 2017).

Table A.1. SMA $230 \mathrm{GHz}$ continuum sensitivity.

\begin{tabular}{ccccc}
\hline \hline Source & $\begin{array}{c}\text { RA } \\
(\mathrm{J} 2000 .)\end{array}$ & $\begin{array}{c}\text { Dec } \\
\text { J2000. }\end{array}$ & $\begin{array}{c}\text { Sensitivity }^{a} \\
1 \sigma(\mathrm{mJy})\end{array}$ & $\begin{array}{c}\text { beam }^{b} \\
(\operatorname{arcsecs})\end{array}$ \\
\hline G10.99-0.08 & $18: 10: 07.00$ & $-19: 27: 52.90$ & 1.0 & $4.0 \times 3.2$ \\
G28.33+0.07 & $18: 42: 46.98$ & $-04: 04: 02.600$ & 1.0 & $3.7 \times 3.4$ \\
\hline
\end{tabular}

Notes. ${ }^{(a)}$ The maps are dominated by emission and therefore the noise estimate is an upper limit. ${ }^{(b)}$ We report the full-width at half-maximum beam (FWHM) of the synthesized beam. 


\section{Appendix B: Outflow search}
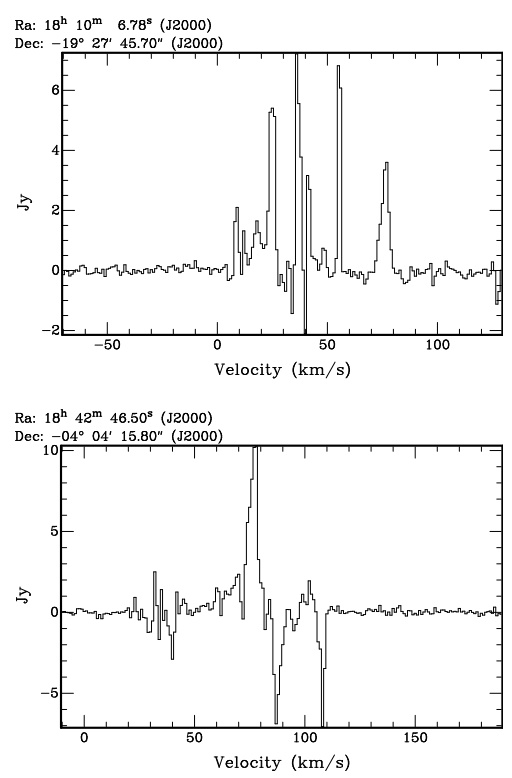

Fig. B.1. CO 2-1 spectrum integrated over a 50 arcsec region around the strong dust continuum peak for G10.99-0.08 (top panel) and $\mathrm{G} 28.33+0.07$ (bottom panel). The velocities extend over $100 \mathrm{~km} \mathrm{~s}^{-1}$. The negative spectral line features likely arise from flux lost in missing spatial components with the SMA.

Table B.1. Outflow upper limits for the main continuum core and integrated over the rest of the map.

\begin{tabular}{|c|c|c|c|}
\hline Feature & $\begin{array}{c}\text { Mass } \\
M_{\odot} \\
\end{array}$ & $\begin{array}{l}\text { Momentum } \\
M_{\odot} \mathrm{km} \mathrm{s}^{-1}\end{array}$ & $\begin{array}{c}\text { Energy } \\
M_{\odot}\left(\mathrm{km} \mathrm{s}^{-1}\right)^{2}\end{array}$ \\
\hline \multicolumn{4}{|c|}{ G10.99-0.08 } \\
\hline Main & $>0.01$ & $>0.05$ & $>0.61$ \\
\hline Rest & $>0.04$ & $>0.64$ & $>12.48$ \\
\hline \multicolumn{4}{|c|}{$\mathrm{G} 28.33+0.07$} \\
\hline Main & $>0.03$ & $>0.37$ & $>5.70$ \\
\hline Rest & $>0.03$ & $>0.40$ & $>5.92$ \\
\hline
\end{tabular}
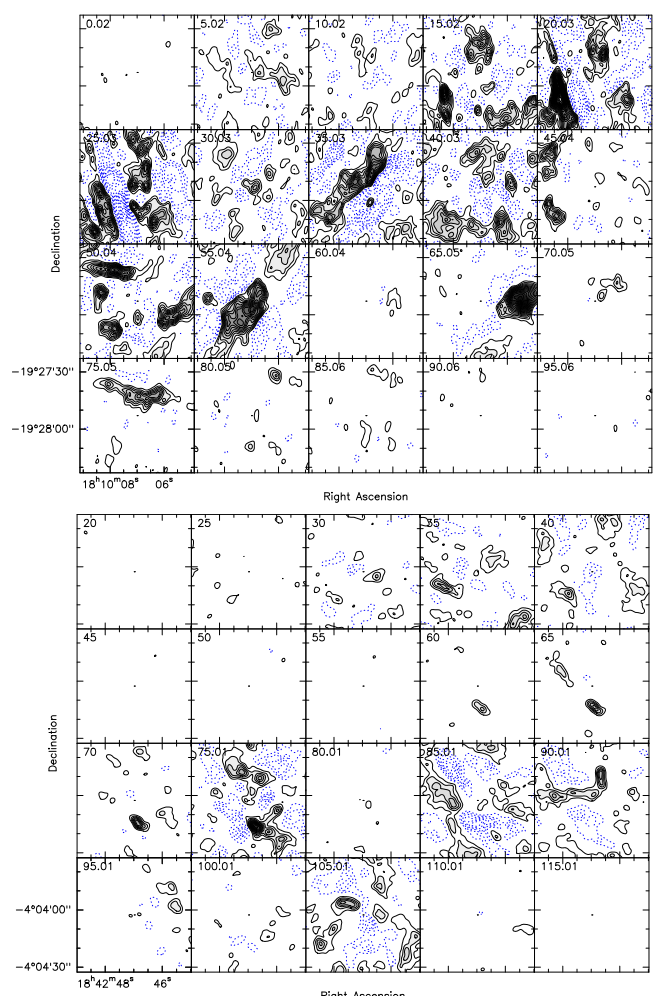

Fig. B.2. CO 2-1 channel maps. These images are the result of averaging the emission over a velocity interval of $5 \mathrm{~km} \mathrm{~s}^{-1}$. The central velocity is labeled on each panel. The contours start at $3 \sigma$ in steps of $3 \sigma$. No additional $u v$ filtering has been applied to the data. The black circle in each images shows the SMA FOV at $230 \mathrm{GHz}$.

Table B.2. SMA $230 \mathrm{GHz}$ continuum fluxes and masses for all dendrogram identified structures.

\begin{tabular}{cccccc}
\hline $\begin{array}{c}\text { RA } \\
(\mathrm{J} 2000)\end{array}$ & $\begin{array}{c}\text { Dec } \\
(\mathrm{J} 2000)\end{array}$ & $\begin{array}{c}\text { Peak flux } \\
\text { mJy beam }^{-1}\end{array}$ & $\begin{array}{c}\text { Flux } \\
\text { Jy }\end{array}$ & $\begin{array}{c}\text { Radius } \\
{ }^{\prime \prime}\end{array}$ & $\begin{array}{c}\text { Mass } \\
M_{\odot}\end{array}$ \\
\hline \multicolumn{5}{c}{ G10.99-0.08 } \\
18:10:07.8 & $-19: 28: 08.8$ & 8.3 & 0.007 & 1.1 & 2.7 \\
18:10:07.5 & $-19: 28: 02.5$ & 10.0 & 0.024 & 1.9 & 9.0 \\
18:10:07.2 & $-19: 27: 50.6$ & 7.7 & 0.004 & 0.8 & 1.5 \\
18:10:06.8 & $-19: 27: 45.3$ & 13.2 & 0.037 & 2.2 & 13.9 \\
$18: 10: 07.0$ & $-19: 27: 36.7$ & 6.6 & 0.004 & 0.8 & 1.3 \\
\hline & & G28.33+0.07 & & \\
$18: 42: 46.5$ & $-4: 04: 15.6$ & 25.7 & 0.050 & 1.8 & 33.1 \\
$18: 42: 46.9$ & $-4: 04: 07.7$ & 12.7 & 0.021 & 1.5 & 14.0 \\
$18: 42: 47.6$ & $-4: 04: 07.0$ & 7.3 & 0.004 & 0.8 & 2.7 \\
$18: 42: 47.2$ & $-4: 03: 52.6$ & 7.0 & 0.013 & 1.6 & 8.7 \\
\hline
\end{tabular}




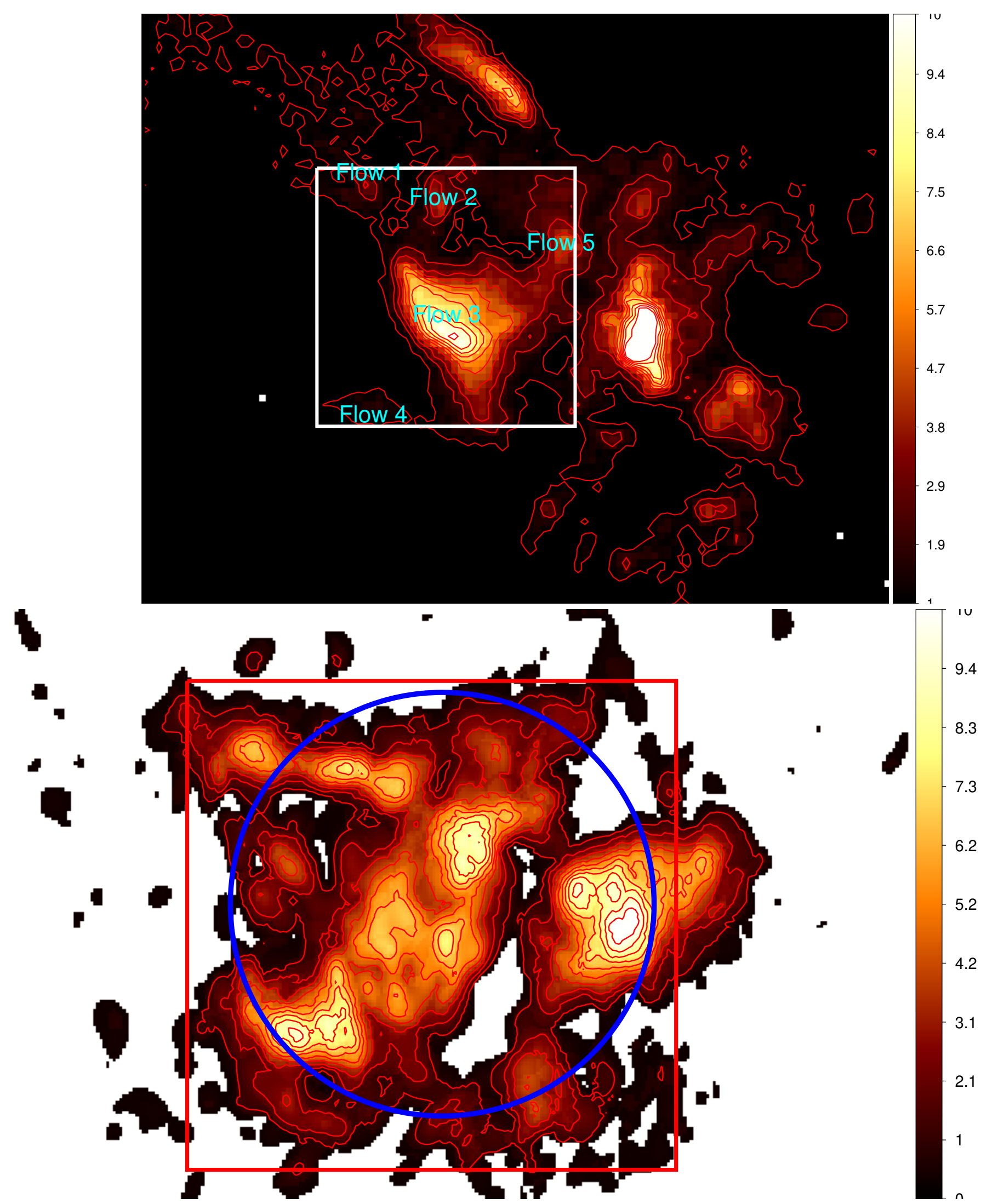

Fig. B.3. Comparison of outflows in NGC 1333 to that observed in G10.99 on same spatial scales. Top panel: CO 1-0 at 1 arcmin resolution integrated intensity from red-shifted $\left(10-15 \mathrm{~km} \mathrm{~s}^{-1}\right)$ for a clustered sub-region in the Perseus NGC 1333 complex scaled to the distance of the G10.99 region $(3.6 \mathrm{kpc})$ (colorscale and contours). Bottom panel: non-primary beam corrected SMA CO 2-1 integrated intensity at $\sim 3$ arcsec resolution for G10.99 for the same spatial scale. In both maps contours start at $1 \mathrm{KKm} \mathrm{s}^{-1}$ up to $10 \mathrm{KKm} \mathrm{s}^{-1}$ in steps of unity and the square indicates the same spatial extent of 1 arcmin. For G10.99 (bottom panel) the blue circle marks the SMA primary beam at $230 \mathrm{GHz}$. Known outflow sources from Arce et al. 2010 within the NGC 1333 region are marked. 